\title{
A Scale Factor Calibration Method for MEMS Gyroscope Based on Virtual Coriolis Force
}

\author{
Yazhou Wang, a, *, Yang Zhao ${ }^{1, ~ b}$, Guoming Xia ${ }^{1, ~ c}$, Anping Qiu ${ }^{1, d}$, Qin Shi ${ }^{1, ~ e}$, Risheng Liu ${ }^{1, f}$, \\ Dan Zhang, g, Jing Wang ${ }^{3, h}$ \\ ${ }^{1}$ Institute of Mechanical Engineering, Nanjing University of Science and Technology, Nanjing \\ 210094, China \\ ${ }^{2}$ Shanghai Institute for space control technology, Shanghai, 200000, China \\ ${ }^{3}$ Sichuan Engineering Technical College, Deyang, 618000, China

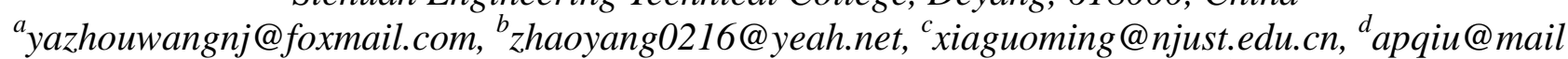 \\ .njust.edu.cn, ${ }^{e}$ sqinhy@njust.edu.cn, $f_{\text {rishliu@qq.com, }{ }^{g} 735554512 @ q q . c o m,{ }^{h} 158034337 @ q q . c o m}$ \\ *Yang Zhao
}

Keywords: calibration, micro gyroscope, virtual Coriolis force, virtual rate-table

Abstract: This paper presents a self-calibration method for a batch of MEMS gyroscopes based on virtual Coriolis force to obtain their scale factor and bandwidth. An electrostatic force is added in the detection axis of the silicon gyroscope to simulate the real Coriolis force to calibrate its scale factor and bandwidth. Thirteen microgyroscopes are tested to verify the feasibility of this method. Compared with traditional method, the proposed method can obtain the scale factor of a bath of gyroscopes with an error less than $0.8 \%$. Besides, the test results show that the measured bandwidth is $354.3 \mathrm{~Hz}$ by the virtual rate-table method with a maximum phase error of $3.39 \mathrm{deg}$, which are in close agreement with the simulation result $349.5 \mathrm{~Hz}$

\section{Introduction}

MEMS gyroscope is an angular rate sensor, which is the core component of inertial navigation and attitude control systems. Compared with conventional rotor gyroscope, electrostatically suspended gyroscope, laser gyroscopes and fiber optic gyroscope, MEMS gyroscope has many advantages, such as small size, light weight, low power consumption, low cost, high reliability and mass production, which make it widely used in industrial control, aerospace, automobile, consumer electronics and military applications

Due to the inevitable error of MEMS process, before putting into practical application the scale factor and bandwidth of the silicon micro gyroscope need to be calibrated. The traditional calibration of the silicon microgyroscope requires complex mechanical equipment, such as rate table and vibration table, which leads to the low efficiency of the calibration, the cost of manpower and material power, and the extension of its $\mathrm{R} \& \mathrm{D}$ cycle $[1,2]$.

Therefore, some calibration techniques without rate-tables are reported in literatures. In [3], a technique using the response to a step input of the mechanical structure to measure the bandwidth was been reported, which relies on establishing a relationship between the step response and the 
frequency response of the gyroscope under test. However, it is more difficult and complex to establish this link as the filter structures. In [4], G. Alexander employed the gravity force to deflect the proof masses, which resulted in a corresponding angular rate input to obtain the scale factor of the gyroscope without rate tables. Because the gravity force of mass can not be changed, it is impossible to complete the calibration within the entire range. In [5], another scale factor calibration method using an additional oscillator to superimpose another vibration with a certain signal can be used as a reference for scale factor calibration. Obviously, the disadvantage is that there must have an additional oscillator added in the mechanical structure. In [6,7], a force electrode was added to the detection axis of the silicon gyroscope, and the static analog angular velocity was applied to calibrate of the scale factor and bandwidth of the silicon micro gyroscope. However, the research on the algorithm for the calibration scheme is not enough.

This paper demonstrates a testing method for scale factor calibration of a batch of MEMS gyroscopes. An electrostatic force is added in the detection axis of the silicon gyroscope to simulate the real Coriolis force to calibrate its scale factor and bandwidth. The scale factor and bandwidth can be determined from the tested dynamic response curve. It provides a convenient and efficient way to calibrate the sensor performance for the microgyroscope operating in closed-loop drive mode and open-loop sense mode, avoiding the disadvantages mentioned above.

\section{Principle Analysis}

MEMS gyroscopes works based on the Coriolis coupling effect, which transfers the energy from the drive mode to the sense mode by inducing the Coriolis force in the sense direction. When the MEMS gyroscopes works, the vibratory in $\mathrm{x}$-direction of the drive mode is keeping constant controlled by system and the vibratory frequency equal to the resonant frequency of drive mode. There is the angular rate of $\Omega_{z}$ in z-direction, which will introduce a Coriolis coupling force $F_{c}$ in $\mathrm{y}$-direction. Then the detected displacement in the $\mathrm{y}$-direction can be used to calculate the angular rate.

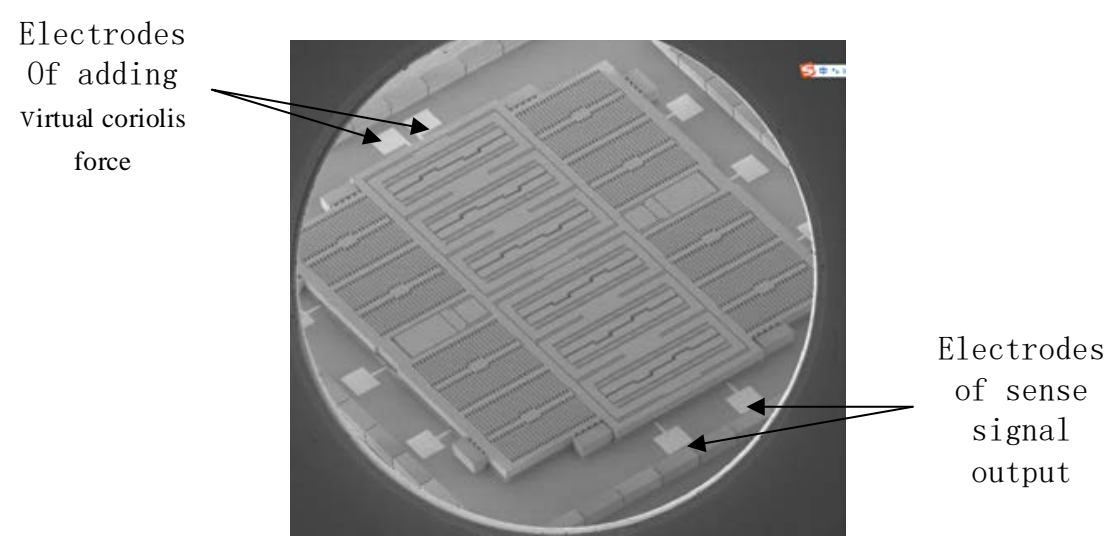

Fig.1 Gyroscope used in the work

Fig 1 shows the signal flow of a gyroscope with the real Coriolis force or the virtual Coriolis force. The $K_{y i}$ and $K_{i v}$ are the conversion coefficients of displacement to current and current to voltage. $\omega_{\mathrm{s}}$ and $Q_{s}$ are the resonant frequency and quality factor of the sense mode. After the readout circuit $\mathrm{A}$ band pass filter $(\mathrm{BPF})$ is set to enhance the signal to-noise ratio. $\omega_{d}$ is the resonant frequency of drive mode. Assume that the amplifiter external angular rate is $\Omega_{z}$, the vibratory amplitude of the drive mode is $x$. Assume that $H=2 x m_{d} \omega_{d} \cos \omega_{d} t$ 


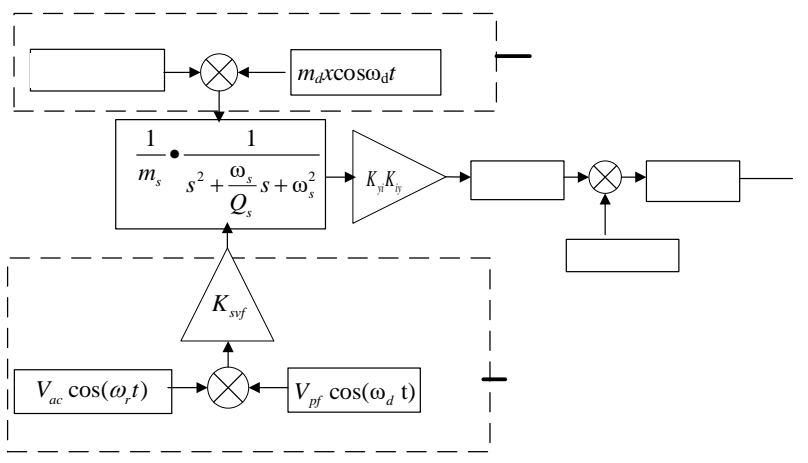

Fig. 2 The signal flow of a gyroscope with the real Coriolis force or the virtual Coriolis force.

The real Coriolis force is given by

$$
F_{\mathrm{c}}=H \Omega_{\mathrm{Z}}\left[\cos \left(\omega_{d}+\omega_{z}\right) \mathrm{t}+\cos \left(\omega_{d}-\omega_{z}\right) \mathrm{t}\right]
$$

The Eq1 indicates that the velocity signal is modulated by the rate signal which requires a demodulation operation to extract the final rate signal.

The output of BPF can be expressed as following,

$$
V_{B P F}=\frac{1}{2} H \Omega_{Z} K_{\mathrm{yi}}\left\{k_{1} \cos \left[\left(\omega_{d}+\omega_{z}\right) t-\varphi_{1}\right]+k_{2} \cos \left[\left(\omega_{d}-\omega_{z}\right) t-\varphi_{2}\right]\right\}
$$

Where, $k_{1}$ and $k_{2}$ are the amplitude gain of the gyroscope for the frequency components $\omega_{\mathrm{d}}+\omega_{\mathrm{z}}$ and $\omega_{\mathrm{d}}-\omega_{\mathrm{z}}, \theta_{1}$ and $\theta_{2}$ are the corresponding total phase delay. Then after demodulated by the reference signal and passing it through a low pass filter (LPF), the final output $V_{0}$ can be expressed as

$$
V_{o}=\frac{1}{4} H \Omega_{Z} K_{\mathrm{o}} \cos \left(\omega_{z} \mathrm{t}-\theta\right)
$$

In which,

$$
\begin{gathered}
K_{\mathrm{o}}=K_{\mathrm{yi}} K_{i v} \sqrt{k_{1}^{2}+k_{2}^{2}+k_{1} k_{2} \cos \left(\varphi_{1}+\varphi_{2}-2 \varphi_{s}\right)} \\
\theta=\arctan \left[\frac{k_{1} \sin \left(\varphi_{1}-\varphi_{s}\right)+k_{2} \sin \left(\varphi_{2}-\varphi_{s}\right)}{k_{1} \cos \left(\varphi_{1}-\varphi_{s}\right)+k_{2} \cos \left(\varphi_{2}-\varphi_{s}\right)}\right] \\
k_{1,2=} \frac{1}{m_{s} \sqrt{\left[\omega_{s}^{2}-\left(\omega_{d} \pm \omega_{z}\right)^{2}\right]^{2}+\left[\frac{\omega_{s}\left(\omega_{d} \pm \omega_{z}\right)}{Q_{s}}\right]^{2}}} \\
\varphi_{s}=\arctan \left[\frac{\omega_{s} \omega_{d}}{\left(\omega_{s}^{2}-\omega_{d}^{2}\right) Q_{s}}\right]
\end{gathered}
$$

Therefore, the frequency response to the angular rate can be obtained as

$$
G\left(\omega_{z}\right)=\frac{1}{4} H K_{\mathrm{o}} \angle \theta
$$

In order to obtain the rate response without rate-tables, an electrostatic force can be constructed 
based on the characteristic of the real Coriolis force and then be applied to the sense mode of microgyroscope instead of the real Coriolis force. $V_{a c} \cos \omega_{d} t$ and $V_{f c} \cos \omega_{z} t$ produced through a signal generator replacing the angular rate and the resonant frequency of the drive mode.

$$
F_{r}=\frac{1}{2} H_{1} V_{a c}\left[\cos \left(\omega_{d}+\omega_{z}\right) t+\cos \left(\omega_{d}-\omega_{z}\right) t\right]
$$

From the fig 1, the process of the real Coriolis force and the virtual Coriolis force is same. Therefore, the frequency response to the virtual angular rate can be obtained as

$$
G_{1}\left(\omega_{z}\right)=\frac{1}{4} H_{1} K_{\mathrm{o}}\left(\omega_{z}\right) \angle \theta
$$

\section{Test and Result}

Table 1 The design value of the tested gyroscopes

\begin{tabular}{|l|l|l|l|}
\hline parameters & $m_{d}(\mathrm{~g})$ & $K_{\text {svf }}(\mathrm{N} / \mathrm{V})$ & $x \omega_{d}(\mathrm{~m} / \mathrm{s})$ \\
\hline value & $9.740 \times 10^{-8}$ & $2.14 \times 10^{-5}$ & 0.206 \\
\hline
\end{tabular}

(a)

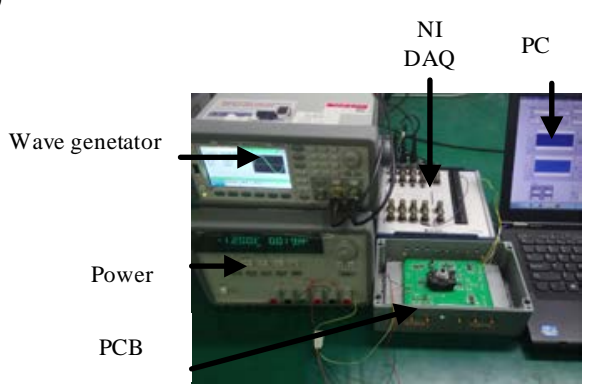

(b)

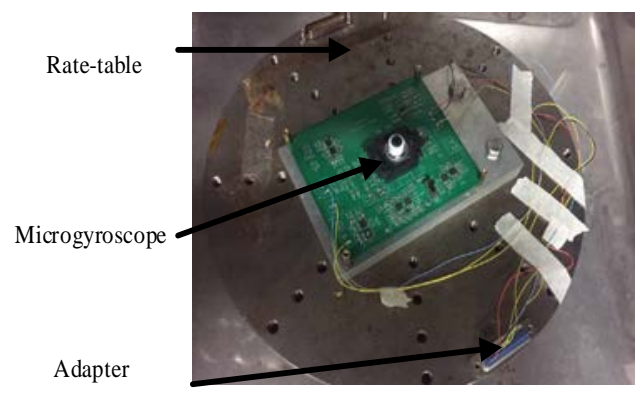

Fig.3 Testing setup of (a) real rate table method and (b) virtual table method

A printed circuit board (PCB) was fabricated to mount with the test circuit and a decoupled Z-axis gyroscope vacuum-sealed in a fixture as shown in Fig.3 (a). The whole control circuit is based on pure analog circuit as shown in Fig.3 (a). The virtual angular rate and virtual vibration velocity are generated by a function waveform generator 33500B with two analog output, respectively. The Coriolis modulation is realized using an analog multiplier AD633, which are then connected to the force electrodes. All signals are transmitted by NI-DAQ6366 to digital signals and sent to PC for analysis and processing.

From the Fig.2, if setting the virtual angular rate as DC component, the VRT method can be used to carry out the static calibration a instead of ordinary rate-table.

The $\Omega_{\mathrm{z}}$ can be replaced by $V_{a c}$ as

$$
\Omega_{Z}=\frac{V_{f c} K_{s v f}}{2 m_{d} x \omega_{d}} \bullet V_{a c}
$$

During the experiment, the value of $V_{f c}$ is $1 \mathrm{~V}$. However, the value of the $K_{s v f}, m_{d}$ and $x$ can not be measured by instrument. In order to build rate-table Virtual Coriolis Force, the design value of them are used. The rate table get by the conventional rate-table method and the virtual rate-table method are as Fig4 (a) and Fig4 (b), respectively. 

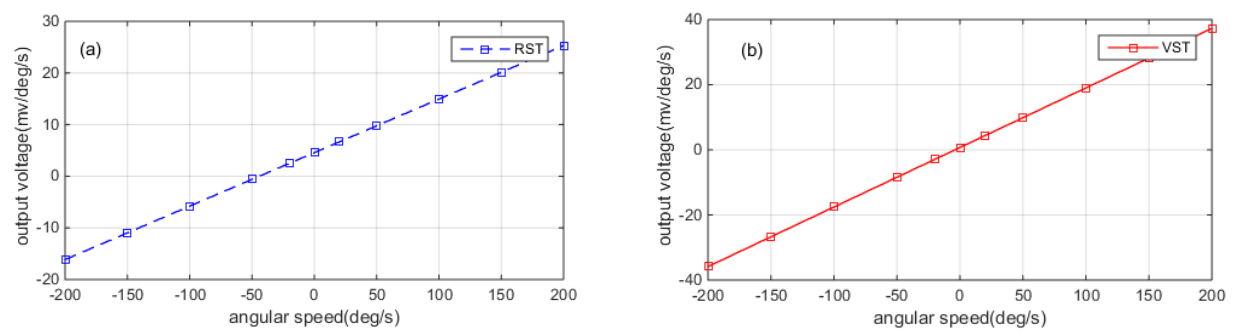

Fig 4. The scale table setting by traditional method (a)andVirtual Coriolis Force method(b)

The test result shows that the conventional rate-table method and the virtual rate-table method are $0.104 \mathrm{mV} / \mathrm{deg} / \mathrm{s}$ and $0.183 \mathrm{mV} / \mathrm{deg} / \mathrm{s}$ with R2 nonlinearity of 287ppm and 189ppm respectively. If we completely rely on the virtual scale factors method without turntable, it is cannot be accepted, obviously. It should be noted that the nonlinearity of them is so small that we gain the rate table by their ratio.

A batch of mirogyroscopes are machined almost simultaneously, the value of the $K_{s v f}, m_{d}$ them mabye very close. The main errors of between the different microgyroscopes are Quality factor and frequency error.

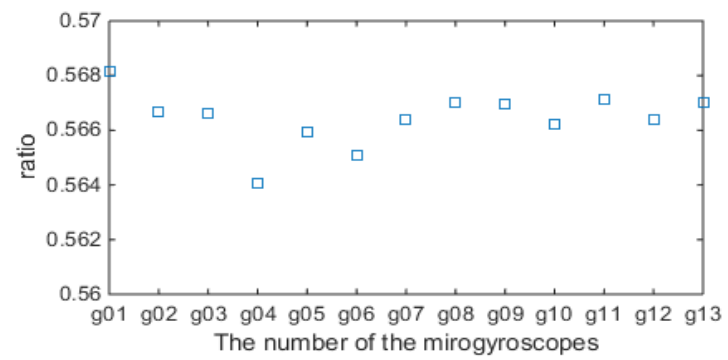

Fig 5 . The ratio of the conventional rate-table method and the virtual rate-table method of 13 mirogyroscopes.

In order to test the accuracy of this method, the ratio be measured with the conventional rate-table method and the virtual rate-table method of 13 mirogyroscopes. The ratio of them are as Fig5

The result shows that biggest difference of each other was $0.79 \%$. So if using one of the ratios to replace the others, the maximum error is less than $0.8 \%$ comparing with traditional method, which is acceptable for calibration.

In order to achieve the dynamic angular rate response of the gyroscope, we can use the AC voltage frequency analog input to complete the dynamic response test when the input angular velocity changes. From the eq4, we can know that The dynamic response of gyroscope is mainly related to $\omega_{d}, Q_{s}$, and $\omega_{s}$. The value of them for tested microgyroscope are in the value of them are in table2. In order to verify the accuracy of this method, a simulation model shown in Fig6 is established. $V_{a c} \cos \left(\omega_{z} t\right)$ and $V_{f c} \cos \left(\omega_{d} t\right)$ are preduced by sinevave block. $\mathrm{M}$ is the gain of sense system, which would be changed by frequency. The second order system is established acconding to the parameters of microgyroscope. BMF is a bandpass filter with pass frequency from $15000 \mathrm{~Hz}$ to $22000 \mathrm{~Hz}$. LMF is a lowpass filter with stop frenquency $2000 \mathrm{~Hz}$. Out1 and out 2 are the real time value of voltage output and virtual angular, which is save to workplace in order to the amplifier and phase delay of them.

Table 2. The parameter of tested microgyroscope

\begin{tabular}{|l|l|l|l|}
\hline parameter & $\omega_{d}(\mathrm{rad} / \mathrm{s})$ & $Q_{S}$ & $\omega_{s}(\mathrm{rad} / \mathrm{s})$ \\
\hline value & 11330 & 9824 & 11715 \\
\hline
\end{tabular}




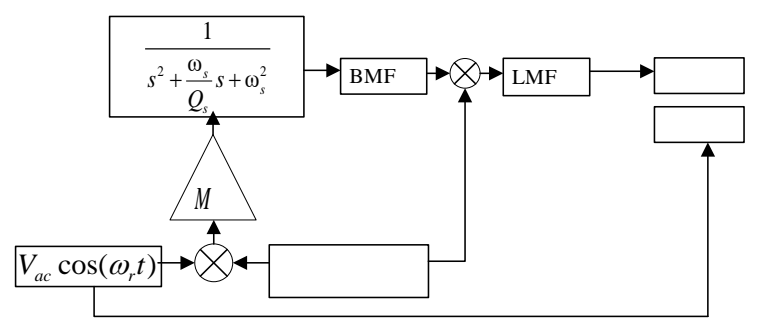

Fig 6. Schematic diagram of Simulation

During the expriment, $V_{f c}=1 \mathrm{~V}, V_{a c}=20 \mathrm{mv}$. For military applications, the microgyroscope bandwidth should be $200 \mathrm{~Hz}$. we measured the dynamic response from $10 \mathrm{~Hz}$ to $500 \mathrm{~Hz}$ for it. The gain is comparing with static frequency respond of microgyroscope got by simulation (SDF) and Virtual Coriolis Force method (VDF) are as Fig 7. The result shows that the bandwidth of VDF are $354.3 \mathrm{~Hz}$, Which is in close agreement with the simulation result $349.5 \mathrm{~Hz}$. The maximum Phase delay error does not exceed 3.39deg at same frequency.
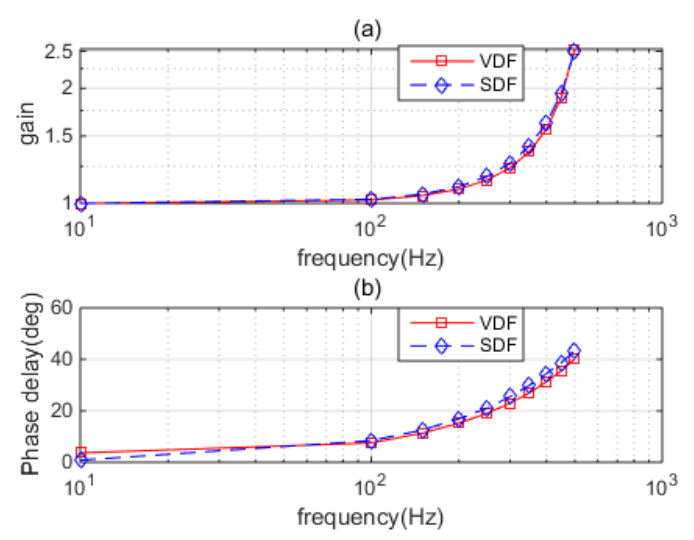

Fig 7. The dynamic response of gyroscope

During the expriment, $V_{f c}=1 \mathrm{~V}, V_{a c}=20 \mathrm{mv}$. For military applications, the microgyroscope bandwidth should be $200 \mathrm{~Hz}$. we measured the dynamic response from $10 \mathrm{~Hz}$ to $500 \mathrm{~Hz}$ for it. The gain is comparing with static frequency respond of microgyroscope got by simulation (SDF) and Virtual Coriolis Force method (VDF) are as Fig 7.

The result shows that the bandwidth of VDF are $354.3 \mathrm{~Hz}$, Which is in close agreement with the simulation result $349.5 \mathrm{~Hz}$. The maximum Phase delay error does not exceed 3.39deg at same frequency.

\section{Conclusion}

A fast calibration method for a batch of MEMS gyroscope based on virtual Coriolis force is demonstrated in this paper. An electrostatic force is added in the detection axis of the silicon gyroscope to simulate the real Coriolis force to calibrate its scale factor and bandwidth. The maximum error is less than $0.8 \%$ comparing with traditional method. The simulation of the frequency response is in good agreement with the experimental results.

\section{Acknowledgments}

This work was supported by the Fundamental Research Funds for the Central Universities, No. $309171 B 8810$. 


\section{References}

[1] Xi Xu and Jie He. Study on New Development and Future Trends of Micromachined Gyroscopes. Piezoelectrics \& Acoustoopics, pp.1004-2074

[2] Lammel G. The future of MEMS sensors in our connected world. 2015 28th IEEE International Conference on. IEEE, pp.61-64

[3] A.A. Trusov,D.DM.Rozelle and A.M.Shkel, 1 ppm precision self-calibrat ion of scale factor in MEMS Coriolis vibratory gyroscopes .Proc.17th Int.Conf. Solid-State Sendors,2013, pp.2531-2534.

[4] A. Gaizer, Z Gao, B Zhou, and Z Chen, Scale factor determination of micro-machined angular rate sensors without a turntable. Tsinghua Sci.Techol, 2006,pp.533-537

[5] T.Link, I. Simon, M. Tradtler and A Gaiser, A new self-test and self-calibration concept formicro-machined gyroscopes Actuat. Microsyst . Dig. Tech. Papers, vol. 1.(2005)

[6] Jimeng Zhang, Chunhua He andYuxian Liu, A Novel Scale Factor Calibration Method for a

MEMS Gyroscope Based on Virtual Coriolis Force. Procedings of the $10^{\text {th }}$ IEEE Interntional Conference on Nano, 2015.

[7] Jian Cui, Chunhua He and Zhenchuan Yuan. Virtual Rate-Table Method for Characterization

of Microgyroscopes. IEEE SENSOR Journal, 2012. 